\title{
GST null polymorphisms may affect the risk of coronary artery disease: evidence from a meta-analysis
}

\author{
Hongling Su*, Yunshan Cao, Jing Li, Yan Zhu and Xuming Ma
}

\begin{abstract}
Background: Whether glutathione S-transferase (GST) null polymorphisms, namely GSTM1 null, GSTP1 null and GSTT1 null polymorphisms, influence the risk of coronary artery disease (CAD) or not remains unclear. Thus, the authors performed a meta-analysis to more robustly estimate associations between GST null polymorphisms and the risk of CAD by integrating the results of previous publications.

Methods: Medline, Embase, Wanfang, VIP and CNKI were searched comprehensively for eligible studies, and 45 genetic association studies were finally selected to be included in this meta-analysis.

Results: We found that GSTM1 null polymorphism was significantly associated with the risk of CAD in overall population ( $\mathrm{OR}=1.37, p=0.003)$ and mixed population $(\mathrm{OR}=1.61, p=0.004)$, GSTP1 null polymorphism was significantly associated with the risk of CAD in overall population ( $O R=1.23, p=0.03)$, whereas GSTT1 null polymorphism was significantly associated with the risk of $C A D$ in overall population $(O R=1.23, p=0.02)$, Caucasians $(\mathrm{OR}=1.23, p=0.02)$ and East Asians $(\mathrm{OR}=1.38, p<0.0001)$.
\end{abstract}

Conclusions: This meta-analysis demonstrated that GSTM1 null, GSTP1 null and GSTT1 null polymorphisms were all significantly associated with an increased risk of CAD.

Keywords: Glutathione S-transferase (GST), Null polymorphisms, Coronary artery disease (CAD), Meta-analysis

\section{Background}

Coronary artery disease (CAD) is featured by stenosis or even occlusion of coronary arteries, and their associated myocardial ischemia or infarction $[1,2]$. The exact cause and pathogenesis of CAD are still nuclear despite extensive researches. Nevertheless, accumulating evidence supports that genetic factors play a crucial part in its development. First, family aggregation of $\mathrm{CAD}$ has been observed extensively, and past twin studies have demonstrated that the heredity grade of CHD can be as high as $50 \%$ [3, 4]. Second, numerous genetic polymorphisms have been found to be associated with an increased risk

\footnotetext{
* Correspondence: suhonglingxxx@163.com

Department of Cardiology, Gansu Provincial People's Hospital, No. 204 of Donggang West Road, Lanzhou 730000, Gansu, China
}

of CAD by previous genetic association studies, and screening of common causal mutations has also been demonstrated to be an efficient way to predict the individual risk of developing $\mathrm{CAD}[5,6]$. Overall, these findings jointly indicate that genetic architecture is important for the occurrence and development of CAD.

Oxidative stress, characterized by accumulation of free radicals, membrane lipid peroxidation and DNA damage, has been found to play a critical role in the pathogenesis of various atherothrombotic disorders including $\mathrm{CAD}[7,8]$. Glutathione-S-transferases (GSTs) are a group of enzymes that play vital roles in regulating cellular detoxification of various exogenous toxins [9]. Moreover, it has been shown that GSTs have anti-oxidation effects and they can protect cells against oxidative stress and its associated DNA 
damage [10]. Previous experimental studies have demonstrated that GST null polymorphisms, which include null polymorphisms of GSTM1 (mu), GSTP1 (pi) and GSTT1 (theta) can result in a diminished gene expression level and a reduced enzymatic activity of GST $[11,12]$. Consequently, it is biologically plausible that GST null polymorphisms may also affect the risk of CAD. Over the last decade, investigators across the world have repeatedly attempted to assess the associations between GST null polymorphisms and the risk of $\mathrm{CAD}$, with inconsistent findings. So a metaanalysis was performed by us to more robustly estimate the associations between GST null polymorphisms and the risk of CAD by integrating the results of previous publications.

\section{Methods}

This meta-analysis was conducted in accordance with the PRISMA guideline [13].

\section{Literature search and inclusion criteria}

Medline, Embase, Wanfang, VIP and CNKI were comprehensively searched by the authors using the below keywords: (glutathione S-transferase OR GST) AND (polymorphism OR polymorphic OR variation OR variant OR mutant OR mutation OR SNP OR genotypic OR genotype OR allelic OR allele) AND (coronary atherosclerotic heart disease OR coronary heart disease OR coronary artery disease OR ischemic heart disease OR angina pectoris OR acute coronary syndrome OR myocardial infarction OR CHD OR CAD OR IHD OR ACS OR MI). Moreover, we also manually screened the references of retrieved publications to make up for the potential incompleteness of literature searching from electronic databases.

Selection criteria of this meta-analysis were listed below: 1. Studies of case-control or cohort design; 2. Give genotypic frequencies of GST null polymorphisms in cases with CAD and population-based controls; 3 . The full manuscript with detailed genotypic frequencies of GST null polymorphisms is retrievable or buyable. Articles would be excluded if one of the following three criteria is satisfied: 1 . Studies without complete genotypic data of GST null polymorphisms in cases with $\mathrm{CAD}$ and population-based controls; 2 . Narrative or systematic reviews, meta-analysis or comments; 3. Case series of subjects with CAD only. If duplicate reports are retrieved, we would only include the most complete one for integrated analyses.

\section{Data extraction and quality assessment}

The authors extracted the following data items from eligible studies: 1 . Last name of the leading author; 2 . Year of publication; 3 . Country and ethnicity of study population; 4. The number of cases with CAD and populationbased controls; 5. Genotypic frequencies of GST null polymorphisms in cases with CAD and population-based controls. The quality of eligible publications was assessed by the Newcastle-Ottawa scale (NOS) [14], and these with a score of 7 - 9 were considered to be of good quality. Two authors extracted data and assessed quality of eligible literatures in parallel. A thorough discussion until a consensus is reached would be endorsed in case of any discrepancy between two authors.

\section{Statistical analyses}

All statistical analyses in this meta-analysis were performed with the Cochrane Review Manager software. Associations between GST null polymorphisms and the risk of CAD were explored by using odds ratio and its 95\% confidence interval. The statistically significant $p$ value was set at 0.05 . The authors used $\mathrm{I}^{2}$ statistics to estimate heterogeneities among included studies. The authors would use DerSimonian-Laird method, which is also known as the random effect model, to integrate the results of eligible studies if $\mathrm{I}^{2}$ is larger than $50 \%$. Otherwise, the authors would use Mantel-Haenszel method, which is also known as the fixed effect model, to integrate the results of eligible studies. Meanwhile, the authors also conduct subgroup analyses by ethnic groups. The overall population (with all study subjects of eligible studies for each polymorphism included) can be divided into Caucasians, Asians or the mixed populations. If the authors specify the ethnic origin of study subjects in their publications, then we would use these data to divide the publications into different subgroups. But if the authors failed to specify the ethnic origin of study subjects in their publications, then we would use the location of the authors' affiliations to divide the publications into different subgroups. For the mixed population, since the authors failed to specify the ethnic origin of study subjects and we could not judge the ethnic origin of study subjects from authors' affiliations neither, it may have several scenarios, which can be a mixture of Caucasians and Africans, a mixture of Caucasians and Asians, a mixture of Africans and Asians, or a mixture of Caucasians, Asians and Africans. Stabilities of integrated results were tested by deleting one study each time, and then integrating the results of the rest of eligible studies. Publication biases were evaluated by assessing symmetry of funnel plots.

\section{Results}

\section{Characteristics of included studies}

One hundred and eighty-four publications were retrieved by using our searching strategy. Among these publications, nine duplicate reports as well as one hundred and four unrelated publications (papers that were not about GST null polymorphisms and the risk of CAD) were omitted, and 71 publications were then selected to screen for eligibility. Seventeen reviews and 
seven case series were further excluded, and another two publications without complete genotypic data were further excluded by the authors. Totally 45 studies met the inclusion criteria, and were finally enrolled for integrated analyses (Fig. 1). The eligible studies were published between 1996 and 2020. Data extracted from eligible studies were summarized in Table 1.

\section{GSTM1 null polymorphism and the risk of CAD}

Thirty-seven studies (17,054 cases and 36,630 controls) assessed relationship between GSTM1 null polymorphism and the risk of CAD. The integrated analyses demonstrated that GSTM1 null polymorphism was significantly associated with the risk of CAD in overall population $(\mathrm{OR}=1.37, p=0.003)$ and mixed population $(\mathrm{OR}=1.61, p=0.004)$ (see Table 2 and Fig. 2 ).

\section{GSTP1 null polymorphism and the risk of CAD}

Eleven studies (4595 cases and 4390 controls) assessed relationship between GSTP1 null polymorphism and the risk of CAD. The integrated analyses demonstrated that GSTP1 null polymorphism was significantly associated with the risk of $C A D$ in overall population $(\mathrm{OR}=1.23$, $p=0.03$ ) (see Table 2 and Fig. 2).

\section{GSTT1 null polymorphism and the risk of CAD}

Thirty-nine studies (17,120 cases and 38,115 controls) assessed relationship between GSTT1 null polymorphism

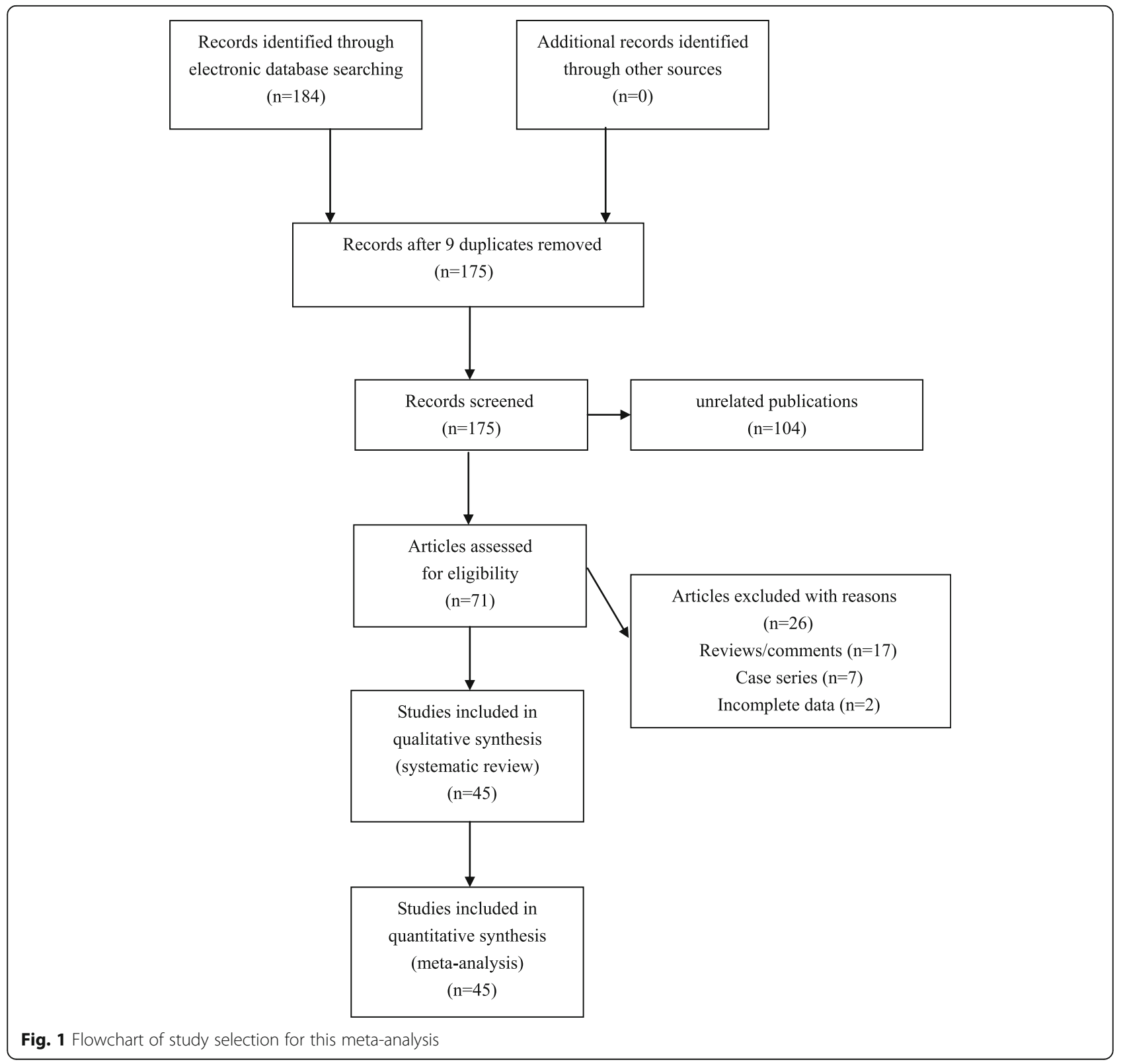


Table 1 The characteristics of included studies in this meta-analysis

\begin{tabular}{|c|c|c|c|c|c|c|c|}
\hline \multirow[t]{2}{*}{ First author, year } & \multirow[t]{2}{*}{ Country } & \multirow[t]{2}{*}{ Ethnicity } & \multirow[t]{2}{*}{ Type of disease } & \multirow{2}{*}{$\begin{array}{l}\text { Sample size } \\
\text { Case/Control }\end{array}$} & \multicolumn{2}{|c|}{ Null genotype [n(\%)] } & \multirow{2}{*}{$\begin{array}{l}\text { NOS } \\
\text { score }\end{array}$} \\
\hline & & & & & Cases Controls & & \\
\hline \multicolumn{8}{|l|}{ GSTM1 null } \\
\hline Abu-Amero 2006 & Saudi Arabia & Mixed & Coronary artery disease (CAD) & $1054 / 762$ & $655(62.1 \%)$ & 117 (15.3\%) & 7 \\
\hline Bazo 2011 & Brazil & Mixed & Coronary artery disease (CAD) & $297 / 96$ & $160(53.8 \%)$ & $40(41.7 \%)$ & 7 \\
\hline Bhat 2016 & India & Mixed & Coronary artery disease (CAD) & $200 / 200$ & $62(31.0 \%)$ & $36(18.0 \%)$ & 8 \\
\hline Bhatti 2018 & India & Mixed & Coronary artery disease (CAD) & $562 / 564$ & 217 (38.6\%) & $127(22.5 \%)$ & 7 \\
\hline Cora 2013 & Turkey & Caucasian & Myocardial infarction (MI) & $324 / 296$ & $182(56.1 \%)$ & $143(48.3 \%)$ & 8 \\
\hline Cornelis 2007 & Canada & Caucasian & Myocardial infarction (MI) & $2042 / 2042$ & $980(48.0 \%)$ & 1041 (51.0\%) & 7 \\
\hline Evans 1996 & Saudi Arabia & Mixed & Coronary artery disease (CAD) & $90 / 884$ & $57(63.3 \%)$ & $484(54.8 \%)$ & 7 \\
\hline Girisha 2004 & India & Mixed & Coronary artery disease (CAD) & 197/198 & $46(23.4 \%)$ & $41(20.7 \%)$ & 7 \\
\hline Hayek 2006 & Israel & Mixed & Coronary artery disease (CAD) & 193/2399 & $88(45.6 \%)$ & $1142(47.6 \%)$ & 8 \\
\hline Kadıoğlu 2016 & Turkey & Caucasian & Coronary artery disease (CAD) & $29 / 30$ & $17(58.6 \%)$ & $14(46.7 \%)$ & 7 \\
\hline Kariž 2012 & Slovenia & Caucasian & Myocardial infarction (MI) & $206 / 257$ & $142(69.0 \%)$ & $166(64.6 \%)$ & 7 \\
\hline Kim 2008 & Korea & East Asian & Coronary artery disease (CAD) & $356 / 336$ & $198(55.6 \%)$ & $191(56.8 \%)$ & 7 \\
\hline Li 2000 & USA & Mixed & Coronary artery disease (CAD) & $400 / 790$ & $178(44.5 \%)$ & $354(44.8 \%)$ & 7 \\
\hline Macie 2009 & Brazil & Mixed & Coronary artery disease (CAD) & $869 / 1573$ & $557(64.1 \%)$ & $789(50.2 \%)$ & 7 \\
\hline Manfredi 2007 & Italy & Caucasian & Coronary artery disease (CAD) & $169 / 53$ & $99(58.6 \%)$ & $24(45.3 \%)$ & 7 \\
\hline Manfredi 2009 & Italy & Caucasian & Coronary artery disease (CAD) & $184 / 47$ & $108(58.7 \%)$ & $18(38.3 \%)$ & 7 \\
\hline Martin 2009 & USA & Mixed & Coronary artery disease (CAD) & $67 / 63$ & $41(61.2 \%)$ & $19(30.2 \%)$ & 7 \\
\hline Masetti 2003 & Italy & Caucasian & Coronary artery disease (CAD) & $308 / 122$ & $163(52.9 \%)$ & $66(54.1 \%)$ & 8 \\
\hline Mir 2016 & India & Mixed & Coronary artery disease (CAD) & $100 / 100$ & $42(42.0 \%)$ & $26(26.0 \%)$ & 8 \\
\hline Nomani 2011 & Iran & Mixed & Coronary artery disease (CAD) & 209/108 & $100(47.8 \%)$ & $57(52.8 \%)$ & 8 \\
\hline Norskov 2011 & Denmark & Caucasian & Coronary artery disease (CAD) & $4930 / 21684$ & $2052(41.6 \%)$ & $11,362(52.4 \%)$ & 7 \\
\hline Olshan 2003 & USA & Mixed & Coronary artery disease (CAD) & $526 / 868$ & $252(47.9 \%)$ & $352(40.6 \%)$ & 8 \\
\hline Pašalić 2017 & Croatia & Caucasian & Coronary artery disease (CAD) & $71 / 174$ & $29(40.8 \%)$ & $69(39.7 \%)$ & 7 \\
\hline Phulukdaree 2012 & India & Mixed & Coronary artery disease (CAD) & $102 / 100$ & $37(36.3 \%)$ & $18(18.0 \%)$ & 7 \\
\hline Pourkeramati 2020 & Iran & Mixed & Coronary artery disease (CAD) & $244 / 281$ & $128(52.5 \%)$ & $138(49.1 \%)$ & 8 \\
\hline Ramprasath 2011 & India & Mixed & Coronary artery disease (CAD) & $290 / 270$ & $128(44.1 \%)$ & $56(20.7 \%)$ & 7 \\
\hline Salama 2002 & USA & Mixed & Coronary artery disease (CAD) & $130 / 90$ & $45(34.6 \%)$ & $33(36.7 \%)$ & 7 \\
\hline Singh 2011 & India & Mixed & Myocardial infarction (MI) & $230 / 300$ & $56(24.3 \%)$ & $65(21.7 \%)$ & 8 \\
\hline Tamer 2004 & Turkey & Caucasian & Coronary artery disease (CAD) & $148 / 247$ & $67(45.3 \%)$ & $103(41.7 \%)$ & 7 \\
\hline Tang 2009 & China & East Asian & Coronary artery disease (CAD) & $277 / 277$ & $89(32,1 \%)$ & $59(21.3 \%)$ & 7 \\
\hline Taspinar 2012 & Turkey & Caucasian & Coronary artery disease (CAD) & $122 / 142$ & $51(41.8 \%)$ & $66(46.5 \%)$ & 7 \\
\hline Wang 2002 & Australia & Caucasian & Coronary artery disease (CAD) & $612 / 256$ & $343(56.0 \%)$ & $153(59.8 \%)$ & 7 \\
\hline Wang 2008 & China & East Asian & Coronary artery disease (CAD) & $277 / 277$ & $89(32.1 \%)$ & $59(21.3 \%)$ & 8 \\
\hline Wilson 2000 & UK & Caucasian & Myocardial infarction (MI) & $356 / 187$ & $191(53.7 \%)$ & $107(57.2 \%)$ & 8 \\
\hline Wilson 2003 & UK & Mixed & Coronary artery disease (CAD) & $170 / 203$ & $70(41.2 \%)$ & $107(52.7 \%)$ & 7 \\
\hline Yeh 2013 & Taiwan & East Asian & Coronary artery disease (CAD) & $458 / 209$ & $253(55.2 \%)$ & $121(57.9 \%)$ & 8 \\
\hline Zhang 2011 & China & East Asian & Coronary artery disease (CAD) & $255 / 145$ & $120(47.1 \%)$ & $46(31.7 \%)$ & 7 \\
\hline \multicolumn{8}{|l|}{ GSTP1 null } \\
\hline Bhat 2016 & India & Mixed & Coronary artery disease (CAD) & $200 / 200$ & $132(66.0 \%)$ & $104(52.0 \%)$ & 8 \\
\hline Bhatti 2018 & India & Mixed & Coronary artery disease (CAD) & $560 / 545$ & $366(65.4 \%)$ & $307(56.3 \%)$ & 7 \\
\hline Cornelis 2007 & Canada & Caucasian & Myocardial infarction (MI) & $2042 / 2042$ & $817(40.0 \%)$ & $817(40.0 \%)$ & 7 \\
\hline Kariž 2012 & Slovenia & Caucasian & Myocardial infarction (MI) & $206 / 257$ & $135(65.5 \%)$ & $140(54.5 \%)$ & 7 \\
\hline
\end{tabular}


Table 1 The characteristics of included studies in this meta-analysis (Continued)

\begin{tabular}{|c|c|c|c|c|c|c|c|}
\hline \multirow[t]{2}{*}{ First author, year } & \multirow[t]{2}{*}{ Country } & \multirow[t]{2}{*}{ Ethnicity } & \multirow[t]{2}{*}{ Type of disease } & \multirow{2}{*}{$\begin{array}{l}\text { Sample size } \\
\text { Case/Control }\end{array}$} & \multicolumn{2}{|c|}{ Null genotype [n(\%)] } & \multirow{2}{*}{$\begin{array}{l}\text { NOS } \\
\text { score }\end{array}$} \\
\hline & & & & & Cases Contro & & \\
\hline Kovacs 2014 & Hungary & Caucasian & Myocardial infarction (MI) & $54 / 78$ & $27(50.0 \%)$ & $26(33.3 \%)$ & 7 \\
\hline Nomani 2011 & Iran & Mixed & Coronary artery disease (CAD) & 209/108 & $118(56.4 \%)$ & $60(55.5 \%)$ & 8 \\
\hline Phulukdaree 2012 & India & Mixed & Coronary artery disease (CAD) & $102 / 100$ & $36(35.3 \%)$ & $52(52.0 \%)$ & 7 \\
\hline Pourkeramati 2020 & Iran & Mixed & Coronary artery disease (CAD) & $244 / 281$ & $64(26.2 \%)$ & $56(19.9 \%)$ & 8 \\
\hline Ramprasath 2011 & India & Mixed & Coronary artery disease (CAD) & $290 / 270$ & $196(67.6 \%)$ & $152(56.3 \%)$ & 7 \\
\hline Singh 2011 & India & Mixed & Myocardial infarction (MI) & $230 / 300$ & $90(39.1 \%)$ & $117(39.0 \%)$ & 8 \\
\hline Yeh 2013 & Taiwan & East Asian & Coronary artery disease (CAD) & $458 / 209$ & $125(27.3 \%)$ & $59(28.2 \%)$ & 8 \\
\hline \multicolumn{8}{|l|}{ GSTT1 null } \\
\hline Abu-Amero 2006 & Saudi Arabia & Mixed & Coronary artery disease (CAD) & $1054 / 762$ & $463(43.9 \%)$ & $66(8.7 \%)$ & 7 \\
\hline Bazo 2011 & Brazil & Mixed & Coronary artery disease (CAD) & $297 / 100$ & $69(23.2 \%)$ & 19 (19.0\%) & 7 \\
\hline Bhat 2016 & India & Mixed & Coronary artery disease (CAD) & $200 / 200$ & $12(6.0 \%)$ & $25(12.5 \%)$ & 8 \\
\hline Bhatti 2018 & India & Mixed & Coronary artery disease (CAD) & $562 / 564$ & $86(15.3 \%)$ & $129(22.9 \%)$ & 7 \\
\hline Cora 2013 & Turkey & Caucasian & Myocardial infarction (MI) & $324 / 296$ & $106(32.7 \%)$ & $63(21.3 \%)$ & 8 \\
\hline Cornelis 2007 & Canada & Caucasian & Myocardial infarction (MI) & $2042 / 2042$ & $388(19.0 \%)$ & $408(20.0 \%)$ & 7 \\
\hline Decharatchakul 2020 & Thailand & East Asian & Coronary artery disease (CAD) & $279 / 735$ & $115(41.9 \%)$ & $242(32.9 \%)$ & 8 \\
\hline García 2018 & Mexico & Mixed & Coronary artery disease (CAD) & $79 / 101$ & $15(19.0 \%)$ & $8(7.9 \%)$ & 7 \\
\hline Girisha 2004 & India & Mixed & Coronary artery disease (CAD) & 197/198 & $15(7.6 \%)$ & $36(18.2 \%)$ & 7 \\
\hline Hayek 2006 & Israel & Mixed & Coronary artery disease (CAD) & 193/2399 & $30(15.5 \%)$ & $392(16.3 \%)$ & 8 \\
\hline Kadıoğlu 2016 & Turkey & Caucasian & Coronary artery disease (CAD) & $29 / 30$ & $6(20.7 \%)$ & $5(16.7 \%)$ & 7 \\
\hline Kariž 2012 & Slovenia & Caucasian & Myocardial infarction (MI) & $206 / 257$ & $77(37.4 \%)$ & $108(42.0 \%)$ & 7 \\
\hline Kim 2008 & Korea & East Asian & Coronary artery disease (CAD) & $356 / 336$ & $196(55.0 \%)$ & $187(55.7 \%)$ & 7 \\
\hline Li 2000 & USA & Mixed & Coronary artery disease (CAD) & $400 / 890$ & $74(18.5 \%)$ & $166(18.7 \%)$ & 7 \\
\hline Lakshmi 2012 & India & Mixed & Coronary artery disease (CAD) & $352 / 282$ & $81(23.0 \%)$ & $39(13.8 \%)$ & 7 \\
\hline Levinsson 2014 & Sweden & Caucasian & Coronary artery disease (CAD) & $112 / 1221$ & $11(9.8 \%)$ & $168(13.8)$ & 7 \\
\hline Macie 2009 & Brazil & Mixed & Coronary artery disease (CAD) & $869 / 1573$ & $209(24.1 \%)$ & $337(21.4 \%)$ & 7 \\
\hline Manfredi 2007 & Italy & Caucasian & Coronary artery disease (CAD) & $169 / 53$ & $95(56.2 \%)$ & $13(24.5 \%)$ & 7 \\
\hline Manfredi 2009 & Italy & Caucasian & Coronary artery disease (CAD) & $184 / 47$ & $84(45.7 \%)$ & $13(27.7 \%)$ & 7 \\
\hline Martin 2009 & USA & Mixed & Coronary artery disease (CAD) & $67 / 63$ & $12(17.9 \%)$ & $12(19.7 \%)$ & 7 \\
\hline Masetti 2003 & Italy & Caucasian & Coronary artery disease (CAD) & $308 / 122$ & $117(38.0 \%)$ & $40(32.8 \%)$ & 8 \\
\hline Mir 2016 & India & Mixed & Coronary artery disease (CAD) & $100 / 100$ & $23(23.0 \%)$ & $16(16.0 \%)$ & 8 \\
\hline Nomani 2011 & Iran & Mixed & Coronary artery disease (CAD) & 209/108 & $16(7.7 \%)$ & $17(15.7 \%)$ & 8 \\
\hline Norskov 2011 & Denmark & Caucasian & Coronary artery disease (CAD) & $4930 / 21684$ & $740(15.0 \%)$ & $3161(14.6 \%)$ & 7 \\
\hline Olshan 2003 & USA & Mixed & Coronary artery disease (CAD) & $526 / 868$ & $75(14.3 \%)$ & $165(19.0 \%)$ & 8 \\
\hline Palmer 2003 & UK & Caucasian & Coronary artery disease (CAD) & $51 / 57$ & $40(78.4 \%)$ & $35(61.4 \%)$ & 7 \\
\hline Pašalić 2017 & Croatia & Caucasian & Coronary artery disease (CAD) & $68 / 177$ & $17(25.0 \%)$ & $54(30.5 \%)$ & 7 \\
\hline Pourkeramati 2020 & Iran & Mixed & Coronary artery disease (CAD) & $244 / 281$ & $129(52.9 \%)$ & $143(50.8 \%)$ & 8 \\
\hline Ramprasath 2011 & India & Mixed & Coronary artery disease (CAD) & 290/492 & $136(46.9 \%)$ & $118(24.0 \%)$ & 7 \\
\hline Salama 2002 & USA & Mixed & Coronary artery disease (CAD) & $130 / 90$ & $32(26.7 \%)$ & $14(15.6 \%)$ & 7 \\
\hline Singh 2011 & India & Mixed & Myocardial infarction (MI) & $230 / 300$ & $23(10.0 \%)$ & $61(20.3 \%)$ & 8 \\
\hline Tamer 2004 & Turkey & Caucasian & Coronary artery disease (CAD) & $148 / 247$ & $48(32.4 \%)$ & $70(28.3 \%)$ & 7 \\
\hline Tang 2009 & China & East Asian & Coronary artery disease (CAD) & $277 / 277$ & $77(27.8 \%)$ & $53(19.1 \%)$ & 7 \\
\hline Taspinar 2012 & Turkey & Caucasian & Coronary artery disease (CAD) & $122 / 142$ & $28(23.0 \%)$ & $25(17.6 \%)$ & 7 \\
\hline Wang 2008 & China & East Asian & Coronary artery disease (CAD) & $277 / 277$ & $77(27.8 \%)$ & $53(19.1 \%)$ & 8 \\
\hline
\end{tabular}


Table 1 The characteristics of included studies in this meta-analysis (Continued)

\begin{tabular}{|c|c|c|c|c|c|c|c|}
\hline \multirow[t]{2}{*}{ First author, year } & \multirow[t]{2}{*}{ Country } & \multirow[t]{2}{*}{ Ethnicity } & \multirow[t]{2}{*}{ Type of disease } & \multirow{2}{*}{$\begin{array}{l}\text { Sample size } \\
\text { Case/Control }\end{array}$} & \multicolumn{2}{|c|}{ Null genotype [n(\%)] } & \multirow{2}{*}{$\begin{array}{l}\text { NOS } \\
\text { score }\end{array}$} \\
\hline & & & & & Cases Contrc & & \\
\hline Wilson 2000 & UK & Caucasian & Myocardial infarction (MI) & $356 / 187$ & $90(25.3 \%)$ & $36(19.3 \%)$ & 8 \\
\hline Wilson 2003 & UK & Mixed & Coronary artery disease (CAD) & $170 / 203$ & $34(20.0 \%)$ & $44(21.7 \%)$ & 7 \\
\hline Yeh 2013 & Taiwan & East Asian & Coronary artery disease (CAD) & $458 / 209$ & $276(60.3 \%)$ & $110(52.6 \%)$ & 8 \\
\hline Zhang 2011 & China & East Asian & Coronary artery disease (CAD) & $255 / 145$ & $141(55.3 \%)$ & $60(41.4 \%)$ & 7 \\
\hline
\end{tabular}

Abbreviations: HWE Hardy-Weinberg equilibrium, NOS Newcastle-Ottawa scale, NA Not available

and the risk of CAD. The integrated analyses demonstrated that GSTT1 null polymorphism was significantly associated with the risk of CAD in overall population $(\mathrm{OR}=1.23, p=$ $0.02)$, Caucasians $(\mathrm{OR}=1.23, p=0.02)$ and East Asians $(\mathrm{OR}=1.38, p<0.0001)$ (see Table 2 and Fig. 2).

\section{Sensitivity analyses}

The authors examined stabilities of integrated analyses results by deleting one study each time, and then integrating the results of the rest of studies. The trends of associations were not significantly altered in sensitivity analyses, which indicated that from statistical perspective, our integrated analyses results were reliable and stable (Relevant datasets can be found at https://osf.io, username: suhonglingxxx@163. com, password: suhonglingxxx@).

\section{Publication biases}

The authors examined potential publication biases in this meta-analysis by assessing symmetry of funnel plots. Funnel plots were found to be generally symmetrical, which indicated that our integrated analyses results were not likely to be severely deteriorated by publication biases (see Fig. 3).

\section{Discussion}

To our knowledge, this is so far the very first metaanalysis regarding associations of GSTM1 and GSTP1 null polymorphisms with the risk of CAD, and this is also so far the most complete meta-analysis regarding GSTT1 null polymorphism and the risk of CAD. The integrated analyses showed that GSTM1 null, GSTP1 null and GSTT1 null polymorphisms were all significantly associated with an increased risk of CAD. Sensitivity analyses suggested that the positive associations observed were quite statistically robust, and no publication bias was detected.

The following points are worth noting when interpreting our integrated findings. Firstly, based on the findings of previous observational studies, we speculated that the investigated GST null polymorphisms may lead to a diminished gene expression level of GST, which may subsequently affect biological functions of GST, result in excessive oxidative stress and ultimately increase the risk of CAD [11, 12]. Secondly, considering that the functional significances of investigated GST null polymorphisms are well established. Our pooled analyses may be still statistically inadequate to detect the actual associations between GST null polymorphisms and CAD in certain ethnic subgroups. Therefore, further studies with larger sample sizes in different populations still

Table 2 Integrated analyses for GST null polymorphisms and CAD

\begin{tabular}{|c|c|c|c|c|c|}
\hline \multirow[t]{2}{*}{ Polymorphisms } & \multirow[t]{2}{*}{ Population } & \multirow{2}{*}{$\begin{array}{l}\text { Sample size } \\
\text { (Cases/ } \\
\text { controls) }\end{array}$} & \multicolumn{3}{|c|}{ Null genotype vs. Present genotype } \\
\hline & & & $P$ value & OR $(95 \% \mathrm{Cl})$ & $\mathrm{I}^{2}$ statistic \\
\hline \multirow[t]{4}{*}{ GSTM1 null } & CAD & $17,054 / 36630$ & 0.003 & $1.37(1.11-1.70)$ & $95 \%$ \\
\hline & Caucasian & $9501 / 25537$ & 0.72 & $1.04(0.85-1.26)$ & $84 \%$ \\
\hline & East Asian & $1623 / 1244$ & 0.07 & $1.35(0.97-1.88)$ & $76 \%$ \\
\hline & Mixed population & $5930 / 9849$ & 0.004 & $1.61(1.16-2.22)$ & $94 \%$ \\
\hline \multirow[t]{3}{*}{ GSTP1 null } & $C A D$ & $4595 / 4390$ & 0.03 & $1.23(1.02-1.48)$ & $70 \%$ \\
\hline & Caucasian & $2302 / 2377$ & 0.17 & $1.35(0.88-2.07)$ & $76 \%$ \\
\hline & Mixed population & 1835/1804 & 0.11 & $1.23(0.96-1.59)$ & $68 \%$ \\
\hline \multirow[t]{4}{*}{ GSTT1 null } & CAD & $17,120 / 38115$ & 0.02 & $1.23(1.03-1.46)$ & $89 \%$ \\
\hline & Caucasian & $9049 / 26562$ & 0.02 & $1.23(1.03-1.47)$ & $67 \%$ \\
\hline & East Asian & 1902/1979 & $<0.0001$ & $1.38(1.20-1.59)$ & $36 \%$ \\
\hline & Mixed population & $6169 / 9574$ & 0.61 & $1.11(0.76-1.62)$ & $94 \%$ \\
\hline
\end{tabular}




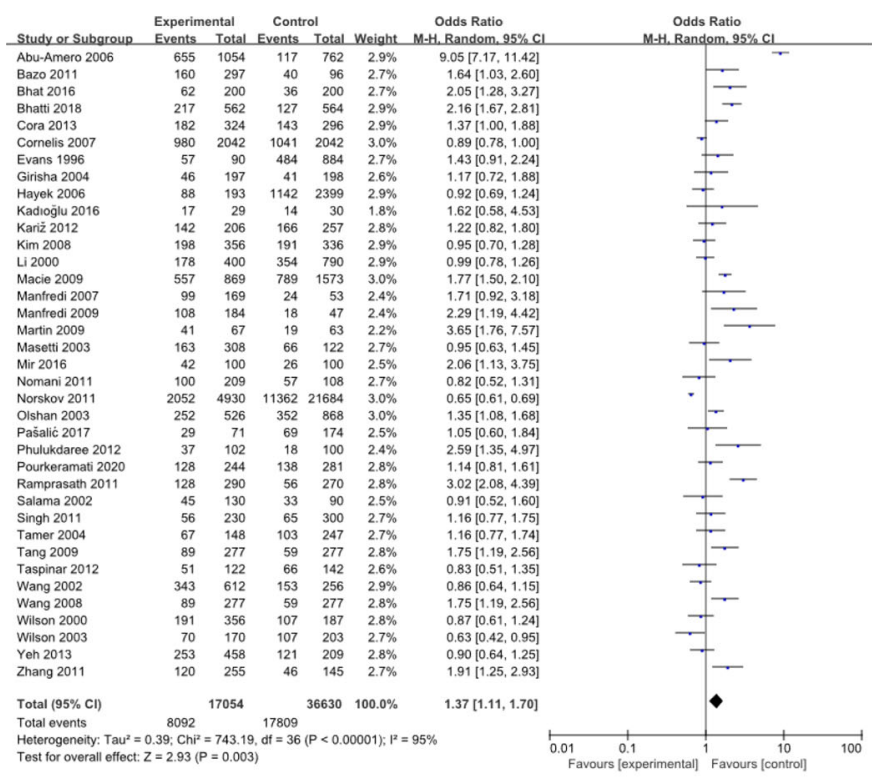

a. Forest plot of GSTM1 null polymorphism and CAD

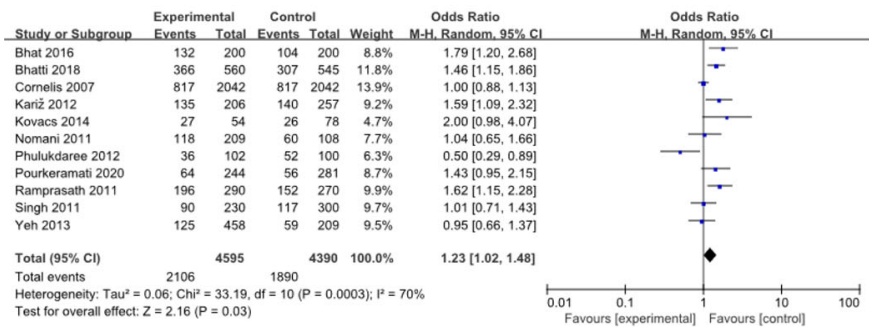

b. Forest plot of GSTP1 null polymorphism and CAD

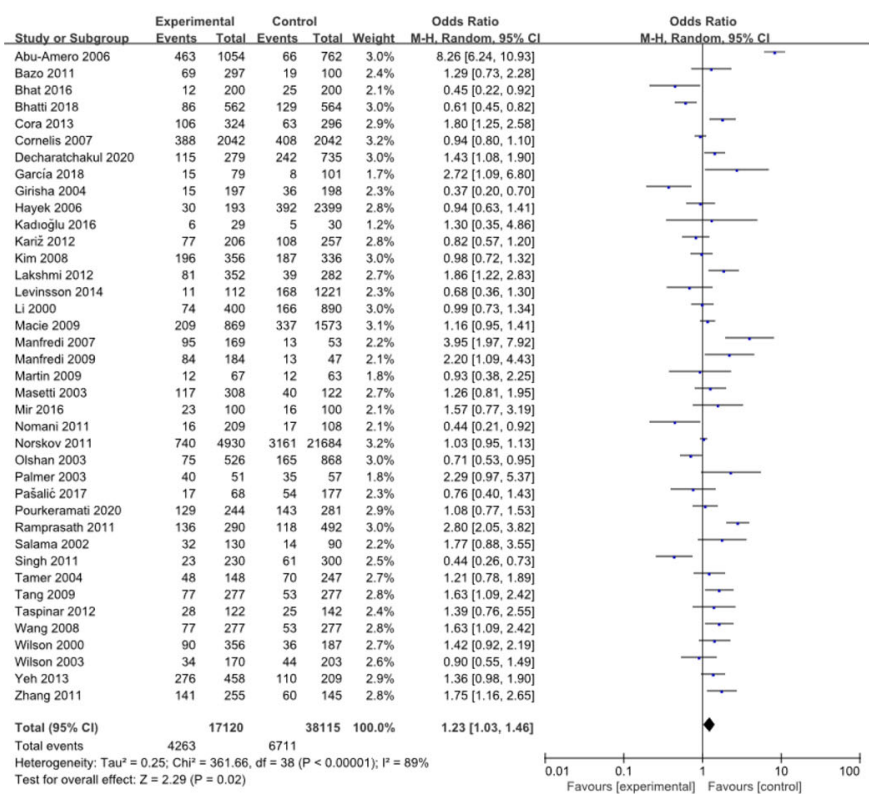

c. Forest plot of GSTTI null polymorphism and CAD

Fig. 2 Forest plots for this meta-analysis 

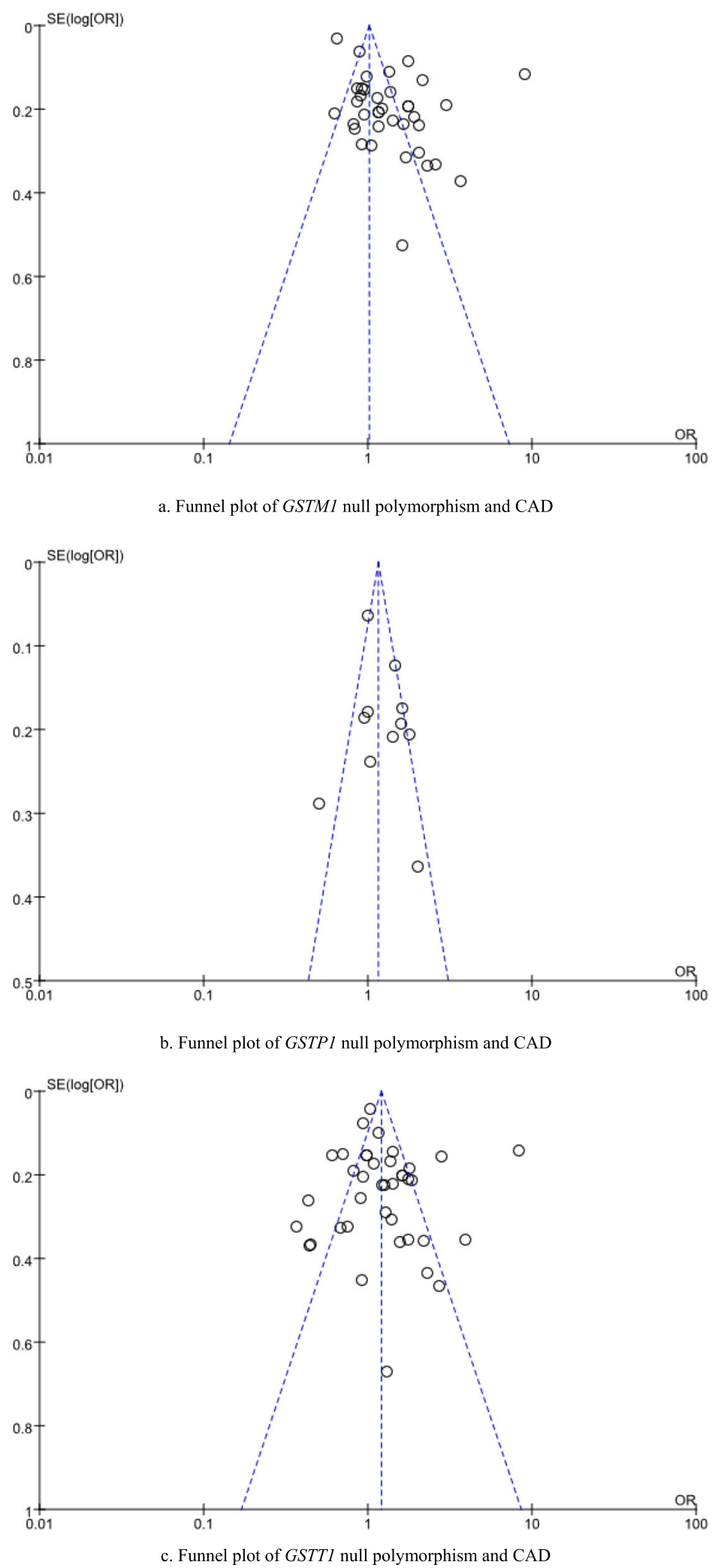

Fig. 3 Funnel plots for this meta-analysis 
need to confirm our findings. Thirdly, we want to study all polymorphic loci of the GST gene initially. Nevertheless, our comprehensive literature searching did not reveal sufficient eligible studies to support integrated analyses for any other polymorphic loci of the GST gene, so we only explored associations with the risk of CAD for three most commonly investigated polymorphisms of the GST gene in this metaanalysis. Fourthly, it is worth noting that previously, Song et al. [15] also tried to investigate associations between GSTT1 null polymorphism and the risk of CAD through a meta-analysis. Nevertheless, this previous meta-analysis only covered relevant genetic association studies that were published before 2014. Since our literature searching revealed that many related studies were published after 2014, an updated meta-analysis like ours is warranted to get more reliable findings. Consistent with the previous meta-analysis, a similar significant finding for GSTT1 null polymorphism was observed in our integrated analyses. Considering that our updated analyses were derived from more eligible studies, our observations should be considered as a valuable confirmation for pre-existing literatures. Fifthly, GST null polymorphisms have also been found to be closely associated with the risk of diabetes, essential hypertension and other types of atherothrombotic disorders such as ischemic stroke or peripheral artery disease [16-20]. Considering that the above mentioned diseases are either considered to be conventional risk factors of $\mathrm{CAD}$ or usually manifest as co-morbid conditions of CAD, it would be interesting to perform some stratified analyses accordingly. Nevertheless, due to the fact that the vast majority of eligible studies failed to report genotypic data according to co-morbid conditions, it is impossible for us to conduct such analyses, and we highly recommend future genetic association studies to carry out stratified analyses according to the co-morbid status of these diseases.

The major limitations of our integrated analyses were listed below. Firstly, our integrated analyses results were derived from unadjusted pooling of previous studies. Without access to raw data of eligible studies, we can only assess associations between GST null polymorphisms and the risk of CAD based on re-calculations of raw genotypic frequencies provided by eligible studies, and we need to admit that lack of further adjustment for baseline characteristics such as age, gender or co-morbid conditions may possibly influence reliability of our findings [21]. Secondly, environmental factors such as smoking status, eating habits or exercise levels may also influence associations between polymorphisms in GST null polymorphisms and the risk of CAD. However, since most of previous studies only paid attention to genetic associations, it is almost impossible for us to explore genetic-environmental interactions in a metaanalysis based on these previous literatures [22]. Thirdly, we did not select 'grey literatures' that were not formally published in peer-reviewed scientific journals for integrated analyses because these literatures are generally considered to be incomplete and it is almost impossible for us to extract all necessary data items from these literatures or assess their quality through the NOS scale. Nevertheless, since we did not select 'grey literatures' for integrated analyses, despite that funnel plots were found to be overall symmetrical, it should be acknowledged that publication biases still may influence reliability of our integrated analyses results [23].

\section{Conclusion}

In conclusion, this meta-analysis demonstrated that GSTM1 null, GSTP1 null and GSTT1 null polymorphisms were all significantly associated with an increased risk of CAD. These findings suggested that GSTM1 null, GSTP1 null and GSTT1 null polymorphisms may have the potential to serve as genetic biomarkers of CAD and they may be used to identify subjects at higher risk of developing CAD. Further studies with larger sample sizes in different populations are still needed to confirm our findings. Moreover, experimental studies are also warranted to reveal the exact underlying mechanisms of the positive associations observed between above mentioned GST null polymorphisms and the risk of CAD in the future.

\section{Abbreviations}

GST: Glutathione S-transferase; CAD: Coronary artery disease; HWE: HardyWeinberg equilibrium; NOS: Newcastle-Ottawa scale; OR: Odds ratios; Cl: Confidence intervals

\section{Acknowledgments}

None.

\section{Authors' contributions}

HS and $Y C$ conceived and designed this meta-analysis. $Y C$ and $J L$ searched literatures. YZ and XM analyzed data. HS and YC wrote the manuscript. All authors have approved the final manuscript as submitted.

Funding

None.

Availability of data and materials

Not applicable.

Ethics approval and consent to participate

Not applicable.

Consent for publication

Not applicable.

Competing interests

The authors declare that they have no competing interests.

Received: 29 June 2020 Accepted: 20 August 2020

Published online: 01 September 2020

References

1. Moran AE, Forouzanfar MH, Roth GA, Mensah GA, Ezzati M, Flaxman A, Murray CJ, Naghavi M. The global burden of ischemic heart disease in 1990 and 2010: the global burden of disease 2010 study. Circulation. 2014;129:1493-501.

2. Global Burden of Disease Study 2013 Collaborators. Global, regional, and national incidence, prevalence, and years lived with disability for 301 acute and chronic diseases and injuries in 188 countries, 1990-2013: a systematic analysis for the Global Burden of Disease Study 2013. Lancet. 2015;386:743-800. 
3. Mayer B, Erdmann J, Schunkert H. Genetics and heritability of coronary artery disease and myocardial infarction. Clin Res Cardiol. 2007;96:1-7.

4. Evans A, Van Baal GC, McCarron P, DeLange M, Soerensen TI, De Geus EJ, Kyvik K, Pedersen NL, Spector TD, Andrew T, Patterson C, Whitfield JB, Zhu G, Martin NG, Kaprio J, Boomsma DI. The genetics of coronary heart disease: the contribution of twin studies. Twin Res. 2003;6:432-41.

5. Sayols-Baixeras S, Lluís-Ganella C, Lucas G, Elosua R. Pathogenesis of coronary artery disease: focus on genetic risk factors and identification of genetic variants. Appl Clin Genet. 2014;7:15-32.

6. Dai X, Wiernek S, Evans JP, Runge MS. Genetics of coronary artery disease and myocardial infarction. World J Cardiol. 2016;8:1-23.

7. Violi F, Loffredo L, Carnevale R, Pignatelli P, Pastori D. Atherothrombosis and oxidative stress: mechanisms and management in elderly. Antioxid Redox Signal. 2017;27:1083-124.

8. Pignatelli P. Menichelli D, Pastori D, Violi F. Oxidative stress and cardiovascular disease: new insights. Kardiol Pol. 2018;76:713-22.

9. Singh S. Cytoprotective and regulatory functions of glutathione Stransferases in cancer cell proliferation and cell death. Cancer Chemother Pharmacol. 2015;75:1-15.

10. Zhang PY, XU X, Li XC. Cardiovascular diseases: oxidative damage and antioxidant protection. Eur Rev Med Pharmacol Sci. 2014;18:3091-6.

11. Dusinská M, Ficek A, Horská A, Raslová K, Petrovská H, Vallová B, Drlicková M, Wood SG, Stupáková A, Gasparovic J, Bobek P, Nagyová A, Kováciková Z, Blazícek P, Liegebel U, Collins AR. Glutathione S-transferase polymorphisms influence the level of oxidative DNA damage and antioxidant protection in humans. Mutat Res. 2001;482:47-55.

12. Santovito A, Cervella P, Delpero M. Baseline frequency of chromosomal aberrations and sister chromatid exchanges in peripheral blood lymphocytes of healthy individuals living in Turin (North-Western Italy): assessment of the effects of age, sex and GSTs gene polymorphisms on the levels of genomic damage. Ann Hum Biol. 2016;43:269-78.

13. Moher D, Liberati A, Tetzlaff J, Altman DG, PRISMA group. Preferred reporting items for systematic reviews and meta-analyses: the PRISMA statement. Ann Intern Med. 2009;151:264-9.

14. Stang A. Critical evaluation of the Newcastle-Ottawa scale for the assessment of the quality of nonrandomized studies in meta-analyses. Eur J Epidemiol. 2010;25:603-5.

15. Song Y, Shan Z, Luo C, Kang C, Yang Y, He P, Li S, Chen L, Jiang X, Liu L. Glutathione S-Transferase T1 (GSTT1) null polymorphism, smoking, and their interaction in coronary heart disease: a comprehensive meta-analysis. Heart Lung Circ. 2017;26:362-70.

16. Bitarafan F, Khodaeian M, Tabatabaei-Malazy O, Amoli MM. Influence of antioxidants' gene variants on risk of diabetes mellitus and its complications: a systematic review. Minerva Endocrinol. 2019;44:310-25.

17. Abbas S, Raza ST, Chandra A, Rizvi S, Ahmed F, Eba A, Mahdi F. Association of ACE, FABP2 and GST genes polymorphism with essential hypertension risk among a North Indian population. Ann Hum Biol. 2015;42:461-9.

18. Coles BF, Kadlubar FF. Human alpha class glutathione S-transferases: genetic polymorphism, expression, and susceptibility to disease. Methods Enzymol. 2005;401:9-42.

19. Bilgin E, Can Demirdöğen B, Türkanoğlu Özçelik A, Demirkaya Ş, Adalı O. Association analysis of glutathione S-transferase omega-1 and omega-2 genetic polymorphisms and ischemic stroke risk in a Turkish population. Neurol Res. 2019;41:118-24.

20. Maciel SS, Pereira Ada C, Silva GJ, Rodrigues MV, Mill JG, Krieger JE. Association between glutathione S-transferase polymorphisms and triglycerides and HDL-cholesterol. Atherosclerosis. 2009;206:204-8.

21. Ma WQ, Han XQ, Wang X, Wang Y, Zhu Y, Liu NF. Associations between XRCC1 gene polymorphisms and coronary artery disease: a meta-analysis. PLoS One. 2016;11:e0166961.

22. Liu A, Wan A, Feng A, Rui R, Zhou B. ICAM-1 gene rs5498 polymorphism decreases the risk of coronary artery disease. Medicine (Baltimore). 2018;97: e12523.

23. Luo Z, Lu Z, Muhammad I, Chen Y, Chen Q, Zhang J, Song Y. Associations of the MTHFR rs 1801133 polymorphism with coronary artery disease and lipid levels: a systematic review and updated meta-analysis. Lipids Health Dis. 2018;17:191

\section{Publisher's Note}

Springer Nature remains neutral with regard to jurisdictional claims in published maps and institutional affiliations.

\section{Ready to submit your research? Choose BMC and benefit from:}

- fast, convenient online submission

- thorough peer review by experienced researchers in your field

- rapid publication on acceptance

- support for research data, including large and complex data types

- gold Open Access which fosters wider collaboration and increased citations

- maximum visibility for your research: over $100 \mathrm{M}$ website views per year

At $\mathrm{BMC}$, research is always in progress.

Learn more biomedcentral.com/submissions 